\title{
Troponin I Type 1 Measurement
}

National Cancer Institute

\section{Source}

National Cancer Institute. Troponin I Type 1 Measurement. NCI Thesaurus. Code

C135447.

The determination of the amount of troponin I type 1 (slow twitch skeletal muscle) in a biological sample. 